\title{
马来海松酸三甲酯区域选择性格氏加成反应研究
}

\author{
姚贵阳 ${ }^{a} \quad$ 韦京辰 $^{a, b} \quad$ 戴伟龙 $^{a}$ 杨 达 ${ }^{a}$ 潘英明*,a 王恒山 ${ }^{*, a}$ \\ $\left({ }^{a}\right.$ 广西师范大学化学化工学院 药用资源化学与药物分子工程重点实验室＼cjkstart桂林 541004) \\ $\left({ }^{b}\right.$ 桂林医学院 药理学教研室 桂林 541004)
}

\begin{abstract}
摘要 研究了格氏试剂与马来海松酸三甲酯(2)的各官能团的反应活性差异, 获得了 2 个区域选择性加成产物(化合物 3 和 4), 各化合物的结构均经元素分析、NMR 和 MS 表征, 用 COSY, HMQC 和 HMBC 进一步对化合物 3 进行了结构分 析, 并由 X 射线单晶衍射确认了其立体结构. 实验结果表明, 2 上的 3 个甲酯基的位阻影响了加成反应的选择性, 内酯 3 的生成阻止了其进一步生成 TADDOL (tetraaryl-1,3-dioxolane-4,5-dimethanols)类产物, 但反应不影响松香环式原有的 立体结构. 产物 3 和 4 有望作为手性衍生试剂.
\end{abstract}

关键词 马来海松酸三甲酯; 格氏试剂; 二维核磁共振; 区域选择性

\section{Study on the Regioselectivie of Grignard Reagent Addition Reaction of Maleopimaric Acid Trimethyl Ester}

\author{
Yao, Guiyang ${ }^{a}$ \\ Wei, Jingchen ${ }^{a, b}$ \\ Dai, Weilong ${ }^{a}$ \\ Yang, $\mathrm{Da}^{a}$ \\ Pan, Yingming ${ }^{*, a}$ \\ Wang, Hengshan ${ }^{*, a}$
}

$\left({ }^{a}\right.$ Key Laboratory for the Chemistry and Molecular Engineering of Medicinal Resources (Ministry of Education of China), School of Chemistry \& Chemical Engineering of Guangxi Normal University, Guilin 541004)

$\left({ }^{b}\right.$ Department of Pharmacology, Guilin Medical College, Guilin 541004)

\begin{abstract}
Two regioselectivite adducts (compounds $\mathbf{3}$ and 4) were firstly synthesized from the Grignard reagent and maleopimaric acid trimethyl ester (2). The structures of all compounds were characterized by elemental analyses, NMR and MS, and stero-structures of $\mathbf{3}$ were further determined by COSY, HMQC and HMBC. The spatial structure of $\mathbf{3}$ was further confirmed by X-ray single crystal diffraction analysis method. Owing to the addition reaction selectivity of $\mathbf{2}$ was dependent on the steric hindrance of three methyl groups, the TADDOL (tetraaryl-1,3-dioxolane-4,5-dimethanols) product was prevented because of the formation of lactone $\mathbf{3}$, but the stereo-structure of the rosin-ring did not changed. Products of $\mathbf{3}$ and $\mathbf{4}$ are expected to apply as chiral derivatization reagents.
\end{abstract}

Keywords maleopimaric acid trimethyl ester; grignard reagent; 2D NMR; regioselectivity

天然手性化合物具有来源方便和底物适应性广的 特点，因而在不对称合成和手性识别中的应用中发挥重 要的作用. 其中, 酒石酸 TADDOL (tetraaryl-1,3-dioxolane-4,5-dimethanols)类型的手性衍生物作为手性配体已 广泛应用于催化剂和手性识别试 剂 ${ }^{[1 \sim 3]}$, 对多数底 物具有高选择性，但应用情况良好的同类试剂还不多， 寻找新型高立体选择性手性配体仍是一个挑战性的课
题. 松香是我国重要的天然资源，松香酸具有多个构型 稳定且易改造的手性官能团, 其衍生物已应用于催化 ${ }^{[4}$ 7]、手性分离 ${ }^{[8 \sim 12]}$. 马来海松酸作为其重要衍生物已应用 于以上方面的研究, 酒石酸 TADDOL 这类反应尚未有 文献报道. 为寻求简单方法制备天然酒石酸类型的新型 手性配体，我们 ${ }^{[13]}$ 在前期的基础上，开展了松香酸酯的 格氏试剂加成反应研究, 利用苯基格氏试剂与马来海松

\footnotetext{
*E-mail: panym2005@sina.com; wang_hengshan@yahoo.com.cn

Received August 8, 2012; revised September 25, 2012; published online September 26, 2012.

Project supported by the Project 973 (No. 2011CB512005), the National Natural Science Foundation of China (Nos. 81260472, 21101035), the Natural Science Foundation of Guangxi Province (No. 2011GXNSFD018010) and the Project of Ten, Hundred, Thousand Distinguished Talents in New Century of Guangxi Province (No. 2007228).

973 计划前期研究专项(No. 2011CB512005)、国家自然科学基金(Nos. 81260472, 21101035)、广西自然科学基金重点(No. 2011GXNSFD018010)、广西 新世纪十百千人才工程人选(No. 2007228)资助项目.
} 
酸三甲酯的区域选择性加成得到化合物 3 和 4 , 并利用 2D NMR 等波谱法确定了 $\mathbf{3}$ 和 $\mathbf{4}$ 的结构, 化合物 $\mathbf{3}$ 的 X-ray 晶体结构验证了格氏加成的区域选择性, 同时实 验结果表明格氏反应对松香环式原有的立体结构没有 影响, 化合物 3 和 4 有望作为手性衍生试剂.

\section{1 结果与讨论}

具有 $C_{2}$ 轴的手性酒石酸 TADDOL 类化合物作为手 性二醇配体已广泛应用于不对称反应的催化剂和手性 识别试剂中, 而且对多数底物具有高选择性, 但不具 $C_{2}$ 轴芳香取代二醇类化合物研究很少, 如糖类衍生物 等 ${ }^{[14 ~ 17]}$. 最近我们发现马来海松酸衍生手性冠醚在对 多种胺类手性底物的识别具有特殊的底物相容性等特 性 ${ }^{[13,18]}$. 因此, 我们选择松香酸酯的格氏试剂加成反应 研究, 以期获得特殊的马来海松酸衍生物, 可以进一步 应用于手性识别和手性配体研究中.

\section{1 合成方法及可能机理}

考虑马来海松酸具有多个构型稳定, 其上有双键和 3 个酯基等且易改造的官能团等特点, 且其结构上没有 酮羰基, 双键不具格氏加成的高反应活性, 我们希望研 究观察苯基格氏试剂加成反应对酯基的选择性. 从结构 上看, 如果仅考虑位阻影响, 格氏加成反应活性次序应 该是 23 或 24 位酯基, 然后是 21 位的酯基. 而且, 我们 前期对脱氢松香酸甲酯的格氏加成反应结果表明, 21 位 的酯基对格氏试剂加成是相对惰性的, 在升温至 $70{ }^{\circ} \mathrm{C}$, 反应时间 $4 \mathrm{~h}$ 后, 产率仅为 $20 \%$. 因此, 有可能实现对 23 或 24 位酯基的加成, 同时保留 21 位的酯基, 这样就 能从马来海松酸三甲酯一步得到 TADDOL 类化合物.

为了考察苯基格氏试剂与马来海松酸三甲酯 $\mathbf{2}$ 的区
域选择性加成, 我们的实验对反应温度(冰浴～室温)、 格氏试剂的浓度、用量等几个方面考察，由表 1 可以看 出, 在室温和 3 倍量试剂的情况下, 主要产物为化合物 3, 只有少量的化合物 $\mathbf{4}$, 升温后化合物 $\mathbf{4}$ 的产率明显增 加，最高两者可以分别达到 70\%和 25\%. 实验发现苯基 格氏试剂加成反应首先得到化合物 $\mathbf{3}$, 而不是直接生成 23,24 位的二醇, 在反应条件下易发生 24 位醇和 23 位 酯基酯交换关环反应. 生成的内酯 3 对格氏试剂惰性, 紧接着加成发生在 21 位的酯基, 生成化合物 4. 而且在 此基础, 再增加温度等条件下 23 位内酯基也不能再进 行反应, 说明内酯活性最低, 也就是说苯基格氏试剂对 马来海松酸三甲酯的酯基基团的反应活性次序应该是 24 位酯基(其次是 21 位酯), 然后是 23 位的酯基, 内酯 3 生成机理可以推测见图 1.

在以化合物 2 为原料, 在无水四氢呋喃中, 冰浴冷 却下加入苯基溴化镁, 在 $0.5 \mathrm{~h}$ 内, 酯 3 的产率为 $65 \%$. 增加温度至 $78{ }^{\circ} \mathrm{C}$, 延长反应时间, 产物 3 的产率最高 可达 70\%, 而产物 4 产率可增加到 25\%(表 1). 在以化合 物 3 为原料, 直接在 $78{ }^{\circ} \mathrm{C}$ 、反应 $4 \mathrm{~h}$ 的条件下，化合物 4 的产率可以达到 50\%. 实验结果表明苯基格氏试剂对 马来海松酸三甲酯的加成反应的区域选择性主要发生 在最初的 $0.5 \mathrm{~h}$ 内, 随后区域选择性迅速下降. 在反应过 程中, 当室温摚拌 $4 \mathrm{~h}$ 后, TLC 追踪原料消失和产物生成 过程(先经过稀酸淬灭反应, 用乙酸乙酯萃取得有机相, 水洗干燥, 可以看到原料及产物消失和生成的现象来进 行监测反应)，由于产物 $\mathbf{3}$ 和 $\mathbf{4}$ 的 $R_{\mathrm{f}}$ 很接近，因此，可以 通过 PTLC 洗脱出产物混合点, 干燥后测定 NMR, 结果 归纳如表 1 .
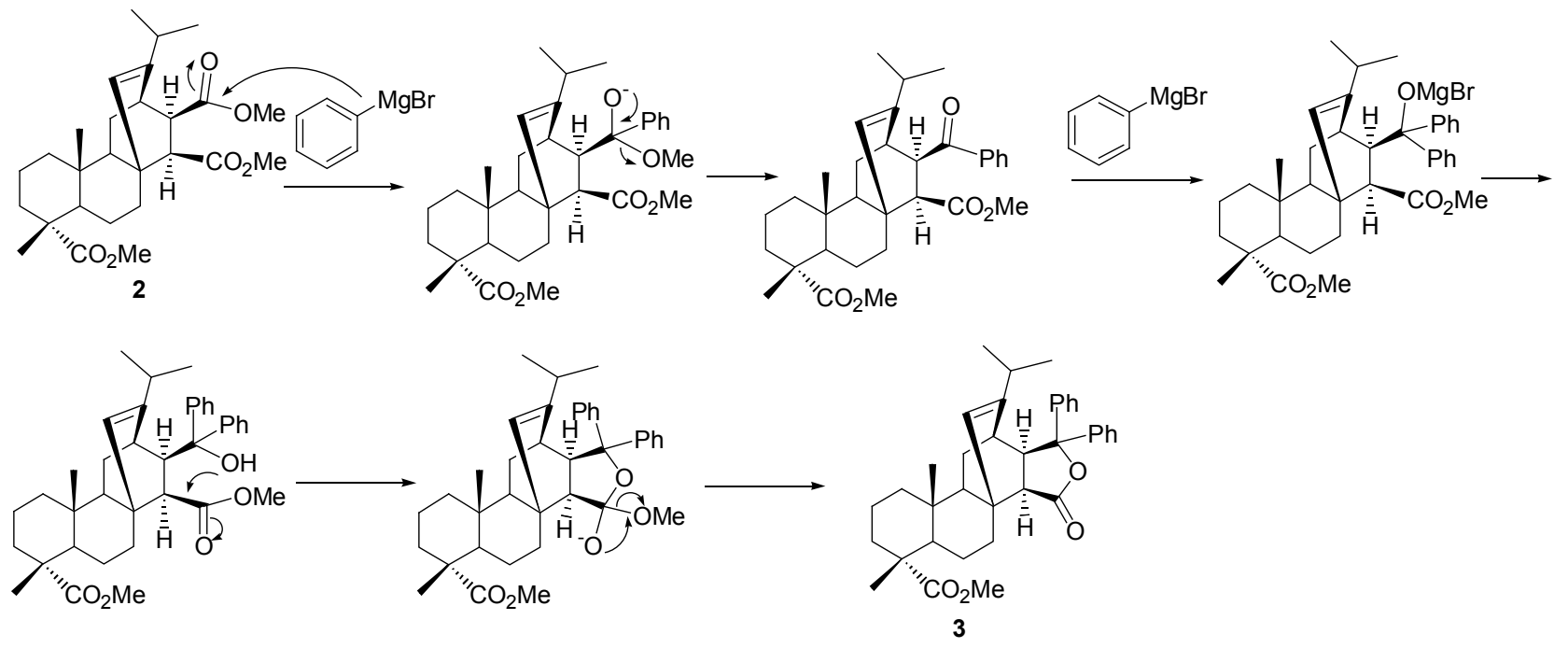

图 1 化合物 $\mathbf{3}$ 的形成机理图

Figure 1 Mechanism of the synthesis of compound 3 
表 1 通过制备薄层色谱和核磁的方法对不同条件下产率的 分析

Table 1 Product yields under different reaction conditions analyzed by HPTLC and NMR

\begin{tabular}{cccccc}
\hline \multirow{2}{*}{ Temp. $/{ }^{\circ} \mathrm{C}$} & \multirow{2}{*}{ Ratio $^{a}$} & \multirow{2}{*}{$t / \mathrm{h}$} & \multicolumn{3}{c}{ Yield/\% of product } \\
\cline { 4 - 6 } & & & 21 位 & 23 位 & 24 位 \\
\hline 25 & 3 & 0.5 & 0 & 65 & 0 \\
78 & 3 & 4 & 20 & 70 & 0 \\
78 & 3 & 6 & 25 & 68 & 0 \\
78 & 3 & 8 & 24 & 65 & 0 \\
\hline${ }^{a} n(\mathrm{PhMgBr}) / n$ (马来海松酸三甲酯). & & &
\end{tabular}

综上所述, 我们是以化合物 2 为原料, 利用马来海 松酸三甲酯中 3 个甲酯的位阻大小的差异进行苯基格氏 试剂的加成, 室温得到化合物 $\mathbf{3}$, 回流可以得到化合物 4. 其中化合物 3 的 21 位酯基水解可以得到新型手性识 别试剂, 其手性识别效果正在研究中.

\subsection{D NMR 谱图和单晶解析}

马来海松酸三甲酯的 24 和 23 位格氏试剂加成产物 是一对区域异构体, 仅用一维 NMR 谱难于确定产物结 构, 为了确定产物的加成位点, 我们利用 HMBC 和 HMQC 对目标物的结构作出进一步的确认.

\subsection{1 ${ }^{1} \mathrm{H}-{ }^{13} \mathrm{C} \mathrm{HMBC}$ 远程偶合谱}

在化合物 3 的 HMBC 谱中可以看到 20-H $(\delta$ 1.15) 与 4-C $(\delta$ 47.13), 5-C $(\delta$ 53.01) 和 21-C $(\delta$ 179.21) 分别是 ${ }^{2} J_{\mathrm{CH}},{ }^{3} J_{\mathrm{CH}}$ 和 ${ }^{3} J_{\mathrm{CH}}$ 相关, $22-\mathrm{H}(\delta 0.56)$ 与 $10-\mathrm{C}(\delta 37.58)$ 相 关, 另外 $\delta=5.49$ 的 $15-\mathrm{H}$ 分别与 $\delta 40.09$ 的 8-C 和 $\delta 36.83$ 的 12-C 相关, $\delta 147.34$ 的 16-C 与 $\delta 0.76$ 的 $17-\mathrm{H}$ 和 $\delta 3.45$ 的 13-H 相关, $\delta 175.76$ 的 23 的 C 与 $\delta 3.45$ 的 $13-\mathrm{H}$ 和 $\delta$ 2.59 的 14- $\mathrm{H}$ 相关; $24-\mathrm{C}(\delta 89.21)$ 分别于 2 '和 $2 "$ 的 $\mathrm{H}$ 相关. 由此可知两个苯环同时加成在 24 位. 所有 ${ }^{1} \mathrm{H}$ 和 ${ }^{13} \mathrm{C}$ 的 远程相关关系见图 2.

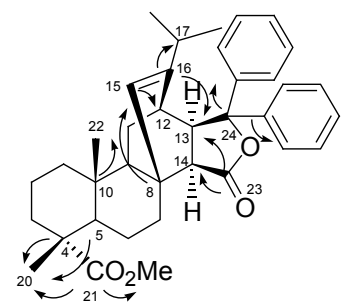

图 2 化合物 3 的 ${ }^{1} \mathrm{H}-{ }^{13} \mathrm{C}$ 远程偶合关系图

Figure $2{ }^{1} \mathrm{H}-{ }^{13} \mathrm{C}$ long-range couple correlation of compound 3

\subsection{2 化合物 3 的单晶结构}

选取 $0.321 \mathrm{~mm} \times 0.28 \mathrm{~mm} \times 0.22 \mathrm{~mm}$ 大小的单晶, 在室温下用 BRUKER Agilent SuperNova 型 X 单晶衍射 仪进行衍射实验. 晶体数据送剑桥晶体中心分配的 CCDC 号码为 898773. 晶体属单斜晶系, $P 2$ (1) 空间群, $a=0.7991 \mathrm{~nm}, \quad b=2.15099 \mathrm{~nm}, c=2.16188 \mathrm{~nm}, \beta=$ $91.145^{\circ}, V=3.30055 \mathrm{~nm}^{3}, Z=2, D=1.169 \mathrm{~g} / \mathrm{cm}^{-3}, \mu=$ $0.079 \mathrm{~mm}^{-1} \cdot F(000)=1276.60$. 采用 $\mathrm{Mo} \mathrm{K \alpha}$ 射线, 石墨 单色器, 方式扫描, 在 $2.40 \leqslant \theta \leqslant 26.00$. 扫描范围内收 集衍射点 11066 个, 其中独立衍射点 8508 , 全部强度数 据经 Lp 因子校正和经验吸收校正，结构由直接法解出, 用最小二乘法对结构参数进行校正, 最终偏离因子 $R=$ $0.0439, w R=0.2735, S=1.04$, 全部计算均用 SHELXS 97 程序完 ${ }^{[19]}$, 图 3 是化合物 3 的晶体结构, 进一步证实 了当 24 位被加成后, 23 位的位阻增大, 进而由于酯交换 而关环。

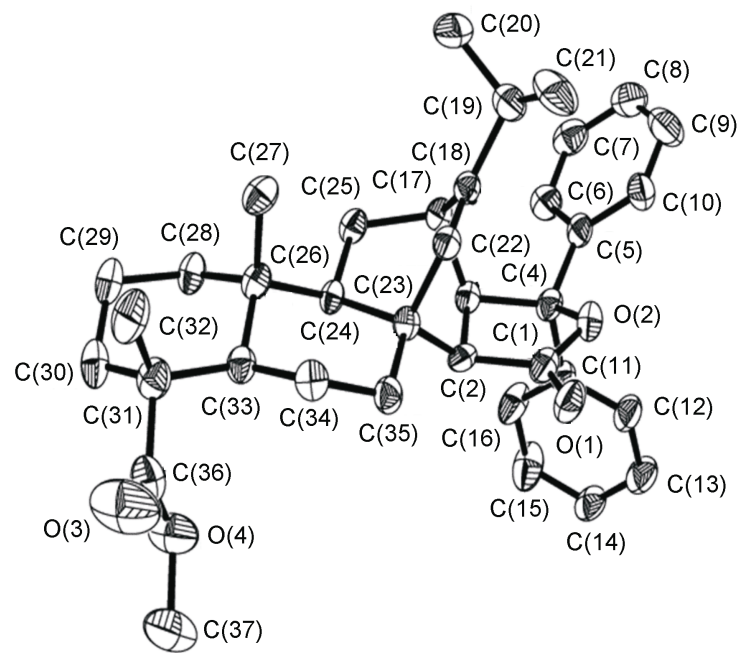

图 3 化合物 $\mathbf{3}$ 的晶体结构图

Figure 3 Molecular structure of compound 3

\section{2 结论}

本文以一级松香为原料经 Diels-Alder 加成反应，酯 化，格氏加成反应获得了 2 个区域选择性加成产物(化 合物 3 和 4). 对化合物 3 的生成机理和格氏试剂对马来 海松酸的区域选择性加成进行了讨论, 并用 X射线确定 了 3 的立体结构, 2 种化合物有望作为手性衍生试剂.

\section{3 实验部分}

\section{1 仪器与试剂}

AVANCE AV 500 超导核磁共振仪(瑞士，布鲁克 公司); Perkin Elmer Model 341 旋光仪(美国, PE 公司); WRS-1A 数字熔点仪 (上海精密科学仪器有限公司); BRUKER ESQUIRE HCT 质谱仪; BRUKER Agilent Super Nova 型 X 单晶衍射仪.

苯基溴化镁, 购自安耐吉化学试剂有限公司; NMR 测定溶剂选用 $\mathrm{CDCl}_{3}$, 百灵威化学试剂有限公司; $\mathrm{PCl}_{3}$ 为分析纯试剂, 新蒸使用, 购自百灵威化学试剂有限公 司；工业一级松香和歧化松香从梧州松脂厂购买，其他 试剂均为分析纯. 


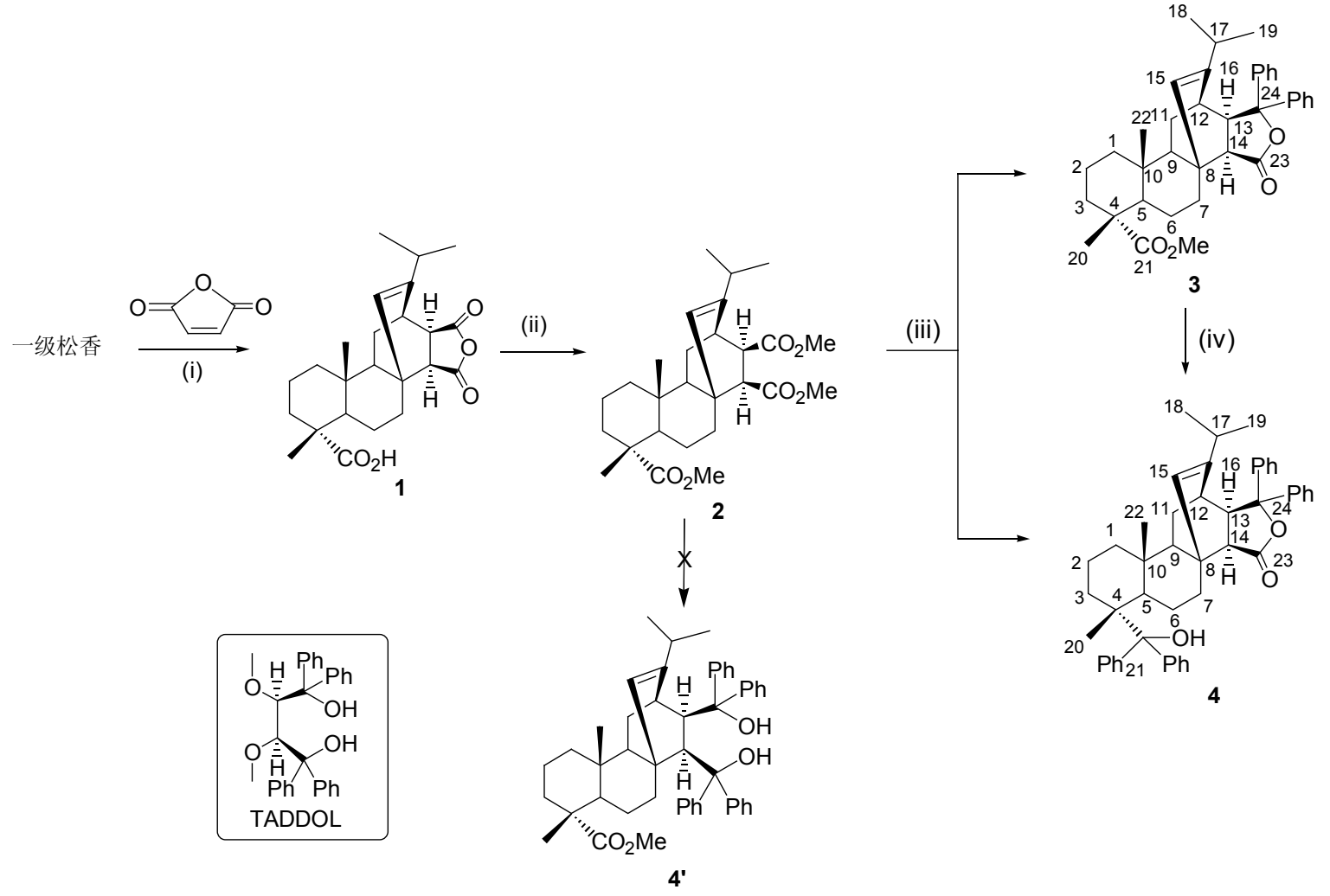

Reagents and conditions: (i) no solvent, $140{ }^{\circ} \mathrm{C}, 3 \mathrm{~h}$; (ii) $\mathrm{PCl}_{3}$, then $\mathrm{MeOH}$, reflux, $4 \mathrm{~h}$; (iii) PhMgBr, THF, r.t., 4 h; (iv) $\mathrm{PhMgBr}$, THF, $78{ }^{\circ} \mathrm{C}, 4 \mathrm{~h}$ Scheme 1

\section{2 实验方法}

\subsection{1 马来海松酸 $\mathbf{1}$ 的合成}

将 $100 \mathrm{~g}$ 磨碎的一级松香置于大烧杯中, 加热升温 至 $140{ }^{\circ} \mathrm{C}$, 不断搅拌下缓慢加入马来酸酐 $32.4 \mathrm{~g}$, 然后 升温至 $170{ }^{\circ} \mathrm{C}$, 持温反应 $3 \mathrm{~h}$, 趁热倒出, 冷却后磨碎, 然后用冰醋酸重结晶 3 次, 过滤, 干燥即得马来海松酸 晶体 ${ }^{[20]}$, 产率 $50.5 \%$, m.p. $228 \sim 230{ }^{\circ} \mathrm{C}$ (文献值 ${ }^{[20]}$ : m.p. $\left.230 \sim 232{ }^{\circ} \mathrm{C}\right),[\alpha]_{\mathrm{D}}^{25}=-27.1(c=1.0, \mathrm{MeOH})$.

\subsection{2 马来海酸酐三甲酯 2 的合成}

在 $50 \mathrm{~mL}$ 圆底烧瓶中加入 $4 \mathrm{~g}(10 \mathrm{mmol})$ 马来海松酸 和 $3 \mathrm{~mL}$ (34.3 mmol) $\mathrm{PCl}_{3}$, 常温下搅拌反应 $4 \mathrm{~h}$ 后, 35 ${ }^{\circ} \mathrm{C}$ 下减压抽除未反应的 $\mathrm{PCl}_{3}$. 然后加入 $30 \mathrm{~mL}$ 无水甲 醇, 回流反应 $3 \mathrm{~h}$, 趁热过滤除去不溶物质, 收集滤液, 蒸去甲醇，用 $95 \%$ 乙醇重结晶得到白色片状晶体 $3.7 \mathrm{~g}$, 产率 $80.4 \%{ }^{[20]}$, m.p. $102 \sim 104{ }^{\circ} \mathrm{C}$ (文献值 ${ }^{[20]}$ : m.p. 103 $\left.104{ }^{\circ} \mathrm{C}\right),[\alpha]_{\mathrm{D}}^{25}+2.0(c=1.0, \mathrm{EtOH}) ;{ }^{1} \mathrm{H} \mathrm{NMR}\left(\mathrm{CDCl}_{3}\right.$, $500 \mathrm{MHz}) \delta: 0.62(\mathrm{~s}, 3 \mathrm{H}), 0.91 \sim 0.93(\mathrm{~m}, 1 \mathrm{H}), 1.05(\mathrm{~d}$, $J=7.0 \mathrm{~Hz}, 3 \mathrm{H}), 1.09$ (d, $J=7.0 \mathrm{~Hz}, 3 \mathrm{H}), 1.15$ (s, 3H), $1.18 \sim 1.20(\mathrm{~m}, 1 \mathrm{H}), 1.32 \sim 1.35(\mathrm{~m}, 2 \mathrm{H}), 1.40 \sim 1.60(\mathrm{~m}$, $7 \mathrm{H}), 1.66 \sim 1.73(\mathrm{~m}, 3 \mathrm{H}), 1.81 \sim 1.83(\mathrm{~m}, 1 \mathrm{H}), 2.63(\mathrm{~d}, J=$ 6.5, $1.2 \mathrm{~Hz}, 1 \mathrm{H}), 2.78(\mathrm{~d}, J=10.8 \mathrm{~Hz}, 1 \mathrm{H}), 2.93 \sim 3.12(\mathrm{~m}$, $2 \mathrm{H}), 3.53(\mathrm{~s}, 3 \mathrm{H}), 3.54(\mathrm{~s}, 3 \mathrm{H}), 3.67(\mathrm{~s}, 3 \mathrm{H}), 5.39(\mathrm{~s}, 1 \mathrm{H})$.

\subsubsection{4,24-二苯基马来海松酸酐单甲酯(3)合成}

将溶解有 $4.6 \mathrm{~g} 2$ 的 $100 \mathrm{~mL}$ 绝对无水四氢呋喃加入 到 $250 \mathrm{~mL}$ 的三颈烧瓶中, 冰浴冷却下加入苯基溴化镁, 室温摚拌 $4 \mathrm{~h}$, 加入 $50 \mathrm{~mL} \mathrm{20 \%}$ 稀 $\mathrm{H}_{2} \mathrm{SO}_{4}$, 用乙酸乙酯萃 取得有机相, 再水洗, 干燥, 以 $V$ (正已烷) : $V$ (四氢呋 喃 $)=8: 1$ 作为洗脱液, 柱层析分离得纯品得白色固体. 用甲醇-三氯甲烷获得单晶 3 , 产率 $65 \%$. m.p. 174 176 ${ }^{\circ} \mathrm{C} ;[\alpha]_{\mathrm{D}}^{25}=-36.5\left(c=0.5, \mathrm{CHCl}_{3}\right) ;{ }^{1} \mathrm{H} \mathrm{NMR}(500 \mathrm{MHz}$, $\left.\mathrm{CDCl}_{3}\right) \delta: 7.46 \sim 7.18(\mathrm{~m}, 10 \mathrm{H}, \mathrm{Ph}-\mathrm{H}), 5.49(\mathrm{~s}, 1 \mathrm{H}, 15-\mathrm{H})$, $3.65(\mathrm{~s}, 3 \mathrm{H}, 21-\mathrm{H}), 3.76 \sim 3.73(\mathrm{~m}, 1 \mathrm{H}, 9-\mathrm{H}), 1.86 \sim 1.84$ (m, 1H, 5-H), $3.42(\mathrm{~d}, J=8.5 \mathrm{~Hz}, 1 \mathrm{H}, 13-\mathrm{H}), 2.86(\mathrm{~d}, J=$ $7.6 \mathrm{~Hz}, 1 \mathrm{H}, 7-\mathrm{Heq}), 2.63$ (s, 1H, 12-H), 2.59 (d, $J=8.7 \mathrm{~Hz}$, 1H, H-14), $1.75 \sim 1.41$ (m, 7H, 3-H, 11-H, 3-H, 7-H, 2-H, 6-H, 1-H), $1.16(\mathrm{~s}, 3 \mathrm{H}, 20-\mathrm{H}), 1.14 \sim 1.06(\mathrm{~m}, 2 \mathrm{H}, 6-\mathrm{H}$, $11-\mathrm{H}), 0.96 \sim 0.98(\mathrm{~m}, 1 \mathrm{H}, 1-\mathrm{H}), 0.85(\mathrm{~d}, J=14.8 \mathrm{~Hz}, 3 \mathrm{H}$, $18-\mathrm{H}), 0.76 \sim 0.78(\mathrm{~m}, 1 \mathrm{H}, 17-\mathrm{H}), 0.62(\mathrm{~d}, J=16.0 \mathrm{~Hz}, 3 \mathrm{H}$, 19-H), 0.56 (s, 3H, 22-H); ${ }^{13} \mathrm{C}$ NMR (125 MHz, $\left.\mathrm{CDCl}_{3}\right) \delta$ : $179.2 \quad(21), \quad 175.7$ (23), 147.3 (16), $145.8 \sim 125.6$ (12× Ph-C), 125.3 (15), 89.2 (24), 55.2 (14), 53.0 (5), 51.8 (OMe), 49.6 (9), 49.5 (13), 47.1 (4), 40.0 (8), 38.4 (1), 37.5 (10), 36.7 (3), 36.3 (12), 35.2 (7), 31.7 (17), 30.0 (11), 21.8 (6), 21.4 (19), 19.3 (18), 17.1 (2), 16.8 (20), 15.5 (22); MS 
(APCI) $m / z: 553(\mathrm{M}+\mathrm{H})^{+}$. Anal. calcd for $\mathrm{C}_{37} \mathrm{H}_{44} \mathrm{O}_{4}: \mathrm{C}$ 80.40, H 8.02, found C 80.55, H 7.96.

3.2.321,21,24,24-四苯基马来海松酸酐单醇(4)合成 iv 路线法: 将溶解有 $5.5 \mathrm{~g} 3$ 的 $50 \mathrm{~mL}$ 绝对无水四氢 呋喃加入到 $100 \mathrm{~mL}$ 的三颈烧瓶中, 冰浴冷却下加入苯 基溴化镁, $78{ }^{\circ} \mathrm{C}$ 搅拌 $4 \mathrm{~h}$, 加入 $20 \mathrm{~mL} \mathrm{20 \%}$ 稀 $\mathrm{H}_{2} \mathrm{SO}_{4}$, 用 乙酸乙酯萃取得有机相, 再水洗, 干燥, 以 $V$ (正己烷) : $V($ 四氢呋喃 $)=7.5: 1$ 作为洗脱液, 柱层析分离得白色 固体，产率为 $50 \%$.

iii 路线法: 将溶解有 $2.3 \mathrm{~g} 2$ 的 $25 \mathrm{~mL}$ 绝对无水四氢 呋喃加入到 $50 \mathrm{~mL}$ 的三颈烧瓶中, 冰浴冷却下加入苯基 溴化镁, $78{ }^{\circ} \mathrm{C}$ 搅拌 $4 \mathrm{~h}$, 加入 $10 \mathrm{~mL} 20 \%$ 稀 $\mathrm{H}_{2} \mathrm{SO}_{4}$, 用乙 酸乙酯萃取得有机相, 再水洗, 干燥, 以 $V$ (正己烷) : $V($ 四氢呋喃 $)=7.5: 1$ 作为洗脱液, 柱层析分离得纯品 得白色固体, 产率为 $25 \%$. m.p. $186 \sim 188{ }^{\circ} \mathrm{C}$; $[\alpha]_{\mathrm{D}}^{25}-$ $27.4\left(c=0.36, \mathrm{CHCl}_{3}\right) ;{ }^{1} \mathrm{H}$ NMR $\left(500 \mathrm{MHz}, \mathrm{CDCl}_{3}\right) \delta$ : $7.83 \sim 7.18(\mathrm{~m}, 20 \mathrm{H}, \mathrm{Ph}-\mathrm{H}), 5.42(\mathrm{~s}, 1 \mathrm{H}, 15-\mathrm{H}), 3.29$ (d, $J=8.4 \mathrm{~Hz}, 1 \mathrm{H}, 13-\mathrm{H}), 2.58$ (s, 1H, 12-H), 2.52 (d, $J=7.8$ $\mathrm{Hz}, 1 \mathrm{H}, 7-\mathrm{H}), 2.26$ (d, J=8.4 Hz, 1H, H-14), $1.64 \sim 1.28$ $(\mathrm{m}, 9 \mathrm{H}), 1.25(\mathrm{~s}, 3 \mathrm{H}, 4-\mathrm{H}), 1.15 \sim 0.83(\mathrm{~m}, 4 \mathrm{H}), 0.76 \sim$ 0.68 (m, 2H), 0.80 (d, $J=6.2 \mathrm{~Hz}, 3 \mathrm{H}, 18-\mathrm{H}), 0.61$ (d, $J=$ $6.8 \mathrm{~Hz}, 3 \mathrm{H}, 19-\mathrm{H}), 0.59$ (s, 3H, 22-H); ${ }^{13} \mathrm{C}$ NMR (125 $\left.\mathrm{MHz}, \mathrm{CDCl}_{3}\right) \delta: 175.8$ (23), 146.9 (16), 146.0 125.5 (24× Ph-C), 125.7 (15), 89.2 (24), 84.7 (21), 55.1 (14), 53.6 (5), 49.7 (9), 49.3 (13), 46.3 (4), 39.5 (8), 39.0 (1), 38.6 (10), 37.3 (3), 36.4 (12), 34.9 (7), 31.7 (17), 30.4 (11), 21.4 (6), 20.4 (19), 19.3 (18), 18.0 (2), 16.9 (20), 15.91 (22); MS (APCI) $m / z: 677(\mathrm{M}+1)^{+}$. Anal. calcd for $\mathrm{C}_{48} \mathrm{H}_{52} \mathrm{~N}_{2} \mathrm{O}_{3}$ : C 85.17, H 7.74, found C 85.01, H 7.87.

\section{References}

[1] Thomas, J. W.; Jolene, E. T. J. Org. Chem. 2000, 65, 5.

[2] Ann, E. L.; Thomas, J. W. Org. Lett. 2006, 8, 2823.

[3] Joseph G. Chirality 2008, $20,5$.

[4] Jiang X. X.; Zhang, Y. F.; Chan A. S. C.; Wang, R. Org. Lett. 2008, $11,153$.

[5] Jiang, X. X.; Zhang, Y. F.; Liu, X.; Zhang, G.; Lai, L. H.; Wu, L. P.; Zhang, J. N.; Wang, R. J. Org. Chem. 2009, 74, 5562.

[6] Jiang, X. X.; Zhang, Y. F.; Wu, L. P.; Zhang, G.; Liu, X.; Zhang, H. L.; Fu, D.; Wang, R. Adv. Synth. Catal. 2009, 351, 2096.

[7] Jiang, X. X.; Zhang, G.; Fu, D.; Cao, Y. M.; Shen, F. F.; Wang, R. Org. Lett. 2010, 12, 1544.

[8] Ye, F. G.; Wang, H. S.; Huang, B. J.; Zhao, S. L. Electrophoresis 2010, 31, 1488.

[9] Wang, H. S.; Zhao, S. L.; He, M.; Zhao, Z. C.; Pan, Y. M.; Liang, Q.J.Sep.Sci. 2007, 30, 2748.

[10] Zhao, S. L.; Wang, H. S.; Pan, Y. M.; He, M.; Zhao, Z. C. J. Chromatogr. A 2007, 1145, 246.

[11] Zhao, S. L.; Wang, H. S.; Zhang, R. C.; Tang, L. D.; Liu, Y. M. Electrophoresis 2006, 27, 17 .

[12] Wang, H. S.; Zhang, R. C.; Zhao, S. L.; Tang, L. D.; Pan, Y. M. Anal. Chim. Acta 2006, 560, 64.

[13] Wang, H. S.; Tian, X. Y.; Yang, D.; Pan, Y. M.; Wu, Q.; He, C. H. Tetrahedron: Asymmetry 2011, 22, 381

[14] Nishi, H.; Kuwahara, Y. J. Biochem. Biophys. Methods 2001, 48, 89.

[15] Nishi, H.; Kuwahara, Y. J. Pharm. Biomed. 2002, 27, 577.

[16] Chen, J.; Du, Y.; Zhu, F.; Chen, B. J. Chromatogr. A 2010, 1217, 7158.

[17] Zaher, M.; Baussanne, I.; Ravelet, C.; Halder, S.; Haroun, M.; Fize, J.; Décout, J.-L.; Peyrin, E. J. Chromatogr. A 2008, 1185, 291.

[18] Wang, H. S.; He, C. H.; Pan, Y. M.; Yao, G. Y.; Wu, Q.; Deng, H. G. J. Inclusion Phenom. Macrocyclic Chem. 2011, 73, 177.

[19] Sheldrick, G. M. SHELXTL, Version 6.10, Bruker AXS Inc., Madison, Wisconsin, USA, 2000.

[20] Pan, Y. M.; Yang, L.; Wang, H. S.; Zhang, R. C.; Zhang, Y. Acta Crystallogr., Sect. E 2006, 62, o5701. 